\title{
Correction to: Imaging Dynamin-Related Protein 1 (Drp1)-Mediated Mitochondrial Fission in Living Cells
}

\section{Felipe Montecinos-Franjola and Rajesh Ramachandran}

\section{Correction to:}

Chapter 16 in: Rajesh Ramachandran (ed.), Dynamin Superfamily GTPases:

Methods and Protocols, Methods in Molecular Biology, vol. 2159,

https://doi.org/10.1007/978-1-0716-0676-6_16

The chapter was inadvertently published with the incorrect unit $\mathrm{mm}$ (millimeters) as "Step size, $\mathrm{mm}$ (z-stack)" instead of $\mu \mathrm{m}$ (micrometers) as "Step size, $\mu \mathrm{m}$ (z-stack)".

The correction has been incorporated by changing the units from "mm" (millimeters) to " $\mu \mathrm{m}$ " (micrometers) and has been updated as "Step size, $\mu \mathrm{m}$ (z-stack)".

\section{Table 1}

Acquisition parameters for the imaging of live cells using the confocal microscope

\begin{tabular}{ll}
\hline Parameter $^{\mathbf{a}}$ & Value \\
\hline Excitation wavelength $(\mathrm{nm})$ & 488 (EGFP), 543 (mCherry) \\
\hline Laser intensity (\%) & $2-10$ \\
\hline Pixel time, $\mu \mathrm{s}$ & $4-12$ \\
\hline Frame size & $512 \times 512$ or $1024 \times 1024$ \\
\hline Digital zoom & $1-5$ \\
\hline Pinhole (confocal aperture), Airy units & $0.8-1.1$ \\
\hline Detector settings & Photon counting (recommended) or analog \\
\hline Step size, $\mu \mathrm{m}(\mathrm{z}$-stack) & $0.1-0.5$ \\
\hline
\end{tabular}

${ }^{a}$ Parameters optimized for acquisition on the Olympus FV1000 microscope

The updated online version of the chapter can be found at https://doi.org/10.1007/978-1-0716-0676-6_16 